\title{
Sphincter Saving Surgeries for Locally Advanced Low Rectal Cancer after Neoadjuvant Chemoradiation
}

\author{
Mohamed A. E. Salem ${ }^{1 *}$, Hamza A. Hamza ${ }^{2}$, Gamal Amira ${ }^{3}$, Abeer E. Ibrahium ${ }^{4}$, Ahmed A. S. Salem ${ }^{1}$ \\ ${ }^{1}$ Surgical Oncology Department, South Egypt Cancer Institute, Assiut University, Assiut, Egypt; ${ }^{2}$ Radiation Oncology Department, \\ South Egypt Cancer Institute, Assiut University, Assiut, Egypt; ${ }^{3}$ Surgical Oncology Department, National Cancer Institute, Cairo \\ University, Cairo, Egypt; ${ }^{4}$ Medical Oncology Department, South Egypt Cancer Institute, Assiut University, Assiut, Egypt. \\ Email: *salem641972@hotmail.com
}

Received May $28^{\text {th }}, 2013$; revised June $28^{\text {th }}, 2013$; accepted July $2^{\text {nd }}, 2013$

Copyright (C) 2013 Mohamed A. E. Salem et al. This is an open access article distributed under the Creative Commons Attribution License, which permits unrestricted use, distribution, and reproduction in any medium, provided the original work is properly cited.

\begin{abstract}
Background: Rectal cancer accounts for the largest distribution within one anatomical region of the large bowel, with approximately one third of all CRC located within the rectum. The Golden standard treatment of primary rectal cancer is curative surgical resection; however, affine balance remains between disease cure and restoration of gastrointestinal continuity. Combined modality has proven efficacy in many malignant tumors with advantage of organ preservation. Methods: Forty nine (49) patients with low rectal carcinoma were included in a prospective study, between Jan 2007 and Jan 2012. Preoperative chemoradiation was administrated to all patients and subjected to different techniques of sphincter saving surgery. Stage I and Stage IV disease at diagnosis were excluded from the study. Results: Forty nine patients were included in the study. 27 (55\%) patients were male and $22(45 \%)$ were female; the age ranges from 23 years to 70 years with the median age 46 years. The main presenting symptoms were bleeding per rectum and tenesmus, Stage II 18 patients $(36.7 \%)$, stage III 31 patients $(63.3 \%)$. Complete clinical and pathological response in 3 patients (6\%), and complete clinical response with only microscopically residual carcinoma in 20 patients (41\%), partial response in 18 patients (36.7\%), and no significant response in 8 patients (16\%) 7 from 8 were mucoid carcinoma. Low anterior resection (LAR) in 22 patients $(44.9 \%)$, Hartman's procedure in 4 patients $(8.1 \%)$, Coloanal pull-through (COP) was done in 19 patients $(38.9 \%)$ and perineal colostomy in 4 patients $(8.1 \%)$. For patients with colo-anal pull-through technique complete dehiscent and retraction observed in 2 cases, Major leakage in one case, stenosis in 4 cases. Conclusion: There is tendency of colorectal cancers to affect younger groups. Most patients presented in advanced stage. Neadjuvant chemo radiation is an excellent tool in sphincter saving surgery. Coloanal pull-through technique is not a widely spread technique for low rectal cancer with good oncological safety and acceptable functional outcome.
\end{abstract}

Keywords: Coloanal Pull-Through; Colo Rectal Cancer; Neoadjuvant Chemoradiation; Low Anterior Resection

\section{Introduction}

Rectal cancer accounts for the largest distribution within one anatomical region of the large bowel, with approximately one third of all CRC located within the rectum. The Golden standard treatment of primary rectal cancer is curative surgical resection; however, a fine balance remains between disease cure and restoration of gastrointestinal continuity. Combined modality has proven efficacy in many malignant tumors with advantage of organ preservation.

Cancers of the distal rectum pose the double problem of local tumor control and sphincter preservation. Abdominoperineal resection (APR), long considered as the

${ }^{*}$ Corresponding author. standard treatment of tumors with a distal edge located up to $6 \mathrm{~cm}$ from the anal verge, provides local control in a substantial majority of cases, but the resulting loss of sphincter function represents a psychological burden for many patients $[1,2]$

Surgery alone has been the standard treatment for patients with adenocarcinoma of the cancer rectum. Despite advances in surgical technique, local recurrence is still a considerable problem [3]. At time of initial presentation, approximately $15 \%$ of the patients diagnosed with rectal cancer have locally advanced unresectable disease $[4,5]$.

A more precise understanding of failure patterns, leading to the acceptance of distal margins of less than 2 $\mathrm{cm}$, and the recent progress in bowel stapling techniques have made coloanal anastomoses feasible after low rectal 
excision. This latter approach is often associated with preoperative radiotherapy (RT), which may be considered to compensate for the limitations of the surgical technique resulting from narrow radial and distal surgical margins [6-8].

Primary end points of this study to:

1) Assess the pathological response after CT based delineation 3DCRT concurrently with fluorouracil;

2) Feasibility of sphenteric preservation after neoadjuvant chemoradiation;

3) Quality of life after different surgical techniques after chemoradiation to preserve anal sphincter.

Secondary end point is disease free survival and OAS.

\section{Patients and Methods}

From Jan 2007 to Jan 2012, 49 consecutive patients with primary cancers involving the distal rectum (up to $6 \mathrm{~cm}$ or less from the anal verge) were treated by preoperative RT, with concomitant chemotherapy, at the South Egypt Cancer Institute, Assiut University, Assiut, Egypt.

The aim of the preoperative chemo-radiation was essentially to achieve down-staging in T2 tumors, while for T3 tumors the aim was both improvement of local control and down-staging.

\subsection{Inclusion Criteria}

Patients had to present histologically confirmed adenocarinoma of the rectum without evidence of distant metastases, and the inferior edge of the tumor had to be located not further than $6 \mathrm{~cm}$ from the anal verge. Only International Union Against Cancer (UICC) T2-T4, N0 or N1 tumors staged were included. The tumor should not involve more than two-thirds of the rectal circumference to be accessible to CXR therapy. Patients with a performance status $\leq 2$ according to the Eastern Cooperative Oncology Group (ECOG) system.

\subsection{Exclusion Criteria}

Previous pelvic irradiation therapy; Previous history of malignant disease; Any other serious illness and/or major organ dys-function; Pregnancy or lactation.

Pretreatment evaluation included physical examination, proctoscopy and/or colonoscopy, abdominal/pelvic computerized tomography (CT) and/or MRI, chest X-ray and serum carcinoembryonic antigen (CEA).

The distance between anal verge and the caudal edge of the tumor was assessed by proctoscopy and/or digital examination.

The preoperative tumor classification was determined using information from the digital examination (including assessment of mobility), CT and/or MRI scan and/or trans-rectal ultrasound, when available. Pretreatment patient characteristics are displayed in Table 1.
Table 1. pretreatment patient characteristics.

\begin{tabular}{cc}
\hline Age range (mean) & $23-70$ Ys (46 Ys) \\
\hline Male/female & $27 / 22$ \\
$\begin{array}{c}\text { Distance from anal verge to the } \\
\text { lower tumor edge (cm) } \\
\text { Mean }\end{array}$ & $2-6 \mathrm{~cm}$ \\
Stage & $4 \mathrm{~cm}$ \\
Stage II (T3-T4,N0) & $18(36.7 \%)$ \\
Stage III ( T2-T4,N1) only & $31(63.3 \%)$ \\
\hline
\end{tabular}

\subsection{Preoperative Therapy}

On conventional simulator using prone position with full bladder which provide maximal displacement of small bowel out of the pelvis. We use lasyer for patient positioning and immobilization. We use CT and at the same isocenteric position, multiple CT cuts at $0.5 \mathrm{~cm}$ interval throughout the entire volume. CT data then transferred to the computer planning system (Version Xio-Release 4.2). At each CT slice, we delineates our target volumes (CTV1, CTV2, CTV3, PTV) CTV1 (peri-rectal, presacral, internal iliac regions) the caudal extent of this elective target volume should be a minimum of $2 \mathrm{~cm}$ caudal to gross disease, including coverage of the entire mesorectum to the pelvic floor. Unless there is radiographic evidence of extension into the ischiorectal fossa, extension of: CTV1 does not need to go more than a 5 millimeters beyond the levator muscles. For very advanced rectal cancers, extending through the mesorectum or the levators, we add $2 \mathrm{~cm}$ margin up to bone wherever the cancer extends beyond the usual compartments.

CTV2: if there is any extension into gynecologic, genitourinary structures or anal canal, the external iliac region should be added. We added $7-8 \mathrm{~mm}$ margin in soft tissue around the external iliac vessels, but one should consider a larger $10+\mathrm{mm}$ margin anterolaterally especially if small vessels or nodes are identified in this area.

CTV3: boost clinical target volume extend to entire mesorectum and presacral region at involved levels, including $2 \mathrm{~cm}$ cephalic and caudal in the mesorectum and $2 \mathrm{~cm}$ on gross tumor within the anorectum.

PTV margin of $0.7 \mathrm{~cm}$ around CTV, except at skin resulting in PTV1, PTV2 and PTV3 around CTV1, CTV2 and CTV3 respectively.

A complete 3D plan done with tissue inhomogeneity correction taking inconsid-eration normal tissue tollerance as the femoral head and neck, and intestine.

We delivered 4500 cGy in 25 fractions to PTV1 and PTV2 then boost 540 cGy in 3 fractions to PTV3.

\section{Chemotherapy}

Laboratory studies: CBC, serum albumin, bilirubin, AST, ALT, ALP, urea, creatinin and CEA before chemotherapy. 
Chemotherapy regimen: 5 -flourouracil $400 \mathrm{mg} / \mathrm{m}^{2}+$ leucovorin $20 \mathrm{mg} / \mathrm{m}^{2}$ D1-4 of first and fifth week of radiation

\subsection{Surgery}

Surgery was performed at a median interval of 25 days (range 20 - 40 days) from completion of chemo radiation. Low anterior resection was done in 22 (44.9\%) patients (9 with coloanal and 13 with colorectal anastomoses), 4 (8.1\%) patients under continent perineal colostomy, 4 (8.1\%) patients under Hartman's procedures and subsequent continuity using surgical stapler and coloanal pull through was done in 19 (38.9\%) patients $(34.7 \%)$ for those patients where low anterior resection is not oncologically save and the only alternative is APR. A temporary transverse colostomy was performed in 10 patients and temporary ileostomy in 5 patients.

\subsubsection{Techniques of Transanal Pull-Through}

The idea for transanal pull through was taken from management of Hirschsprung's Disease. In patients undergoing transanal pull through with coloanal anastomosis, the technique was standardized according to the following steps: The left colon with the splenic flexure was mobilized after ligature and section of the inferior mesenteric vein and artery. The mesosigmoid was then mobilized and the plane of the mesorectum was entered in continuity as the dissection proceeded distally (Figure 1). This plane was followed down to the level of the pelvic floor when a total mesorectal excision (TME) was performed [9]. The dissection of the rectum and mesorectum was performed applying slight lateral traction to open the cleavage plane between the mesorectum and the lateral wall of the pelvis. This allowed clear visualization and preservation of the pelvic autonomic nerves. Transaction of rectum at least $2 \mathrm{~cm}$ below tumor growth with presservation of anal sphincter, Mucosectomy of any remaining rectal mucosa, telescoping of colon (Figure 2) or ileal loop (Figure 3) through the anus, the colo-anal anastomosis was done from below between colon and the anal canal with $2 / 0$ absorbable sutures, the distal stump leave to be ischemic and trimming done after 7 - 10 days postoperative. Oral feeding started after regain bowel movement.

\subsubsection{Techniques of Continent Perineal Colostomy}

After performing abdominoperineal resection, a $7-8 \mathrm{~cm}$ smooth muscle colonic cuff was prepared from resected segment of the sigmoid colon. Mesenteric fat and epiploic appendages were removed. The colonic tube was then inverted and the mucosa was sharply dissected from the sub mucosa. After a longitudinal incision along tinea coli, a flap 3 to $4 \mathrm{~cm}$ wide and $7 \mathrm{~cm}$ long was created. The neosphincter was created by first securing the flap to

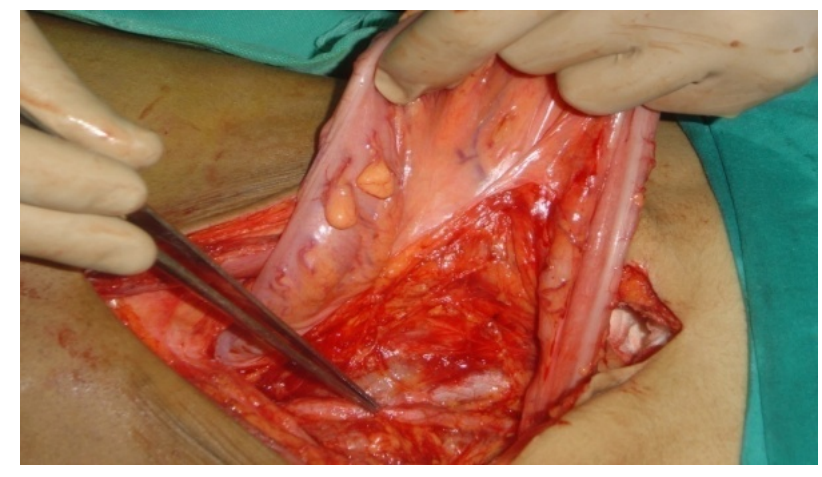

Figure 1. Mesorectum dissection.

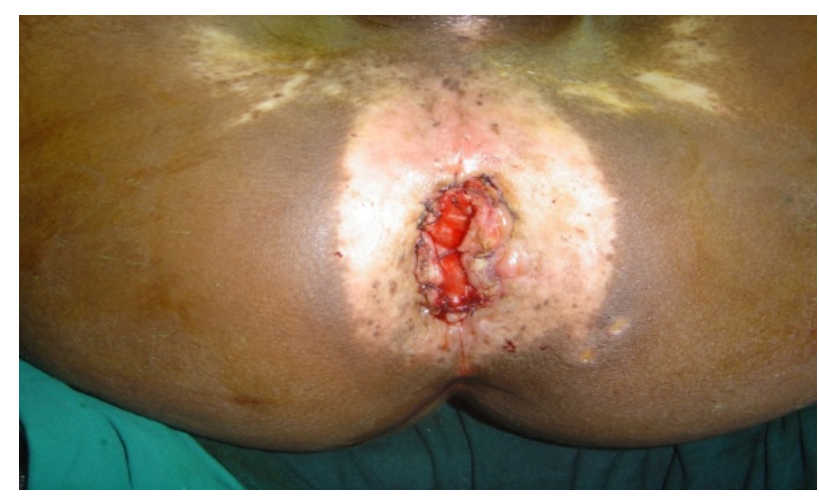

Figure 2. Coloanal pull through.

anti mesenteric portion of colon with interrupted $3-0$ absorbable sutures. This was subsequently wrapped three quarter turns through the rest $3 \mathrm{~cm}$ proximal to terminal colon. The pull-through colon was matured at the skin using interrupted 3 - 0 absorbable sutures in tension free fashion.

\section{Result}

Forty nine (49) patients were included in the study. 27 $(55 \%)$ patients were male and $22(45 \%)$ were female, the age range from 23 years to 70 years with the median age 46 years. The main presenting symptoms were bleeding per rectum and Tenesmus. Stage II eighteen patients (36.7\%) and Stage III thirty one patients (63.3\%).

All patients had a pretreatment biopsy diagnosis of adenocarcinoma (11 well-differentiated, 18 moderately differentiated, 7 poorly differentiated and $13(26.5 \%)$ mucoid carcinoma). Most cases of mucoid carcinoma (10 cases) were in young age under 40 years.

Neoadjuvant chemoradiation was administrated for all patients. pathological evaluation of the resected specimen show all circumferential resection margins were clear by at least $1 \mathrm{~cm}$, complete clinical and pathological response in 3 patients $(6 \%)$, and complete clinical response with only microscopically residual carcinoma in 20 patients (41\%), partial response in 18 patients $(36.7 \%)$, no significant response in 8 patients (16\%) 7 from 8 were 

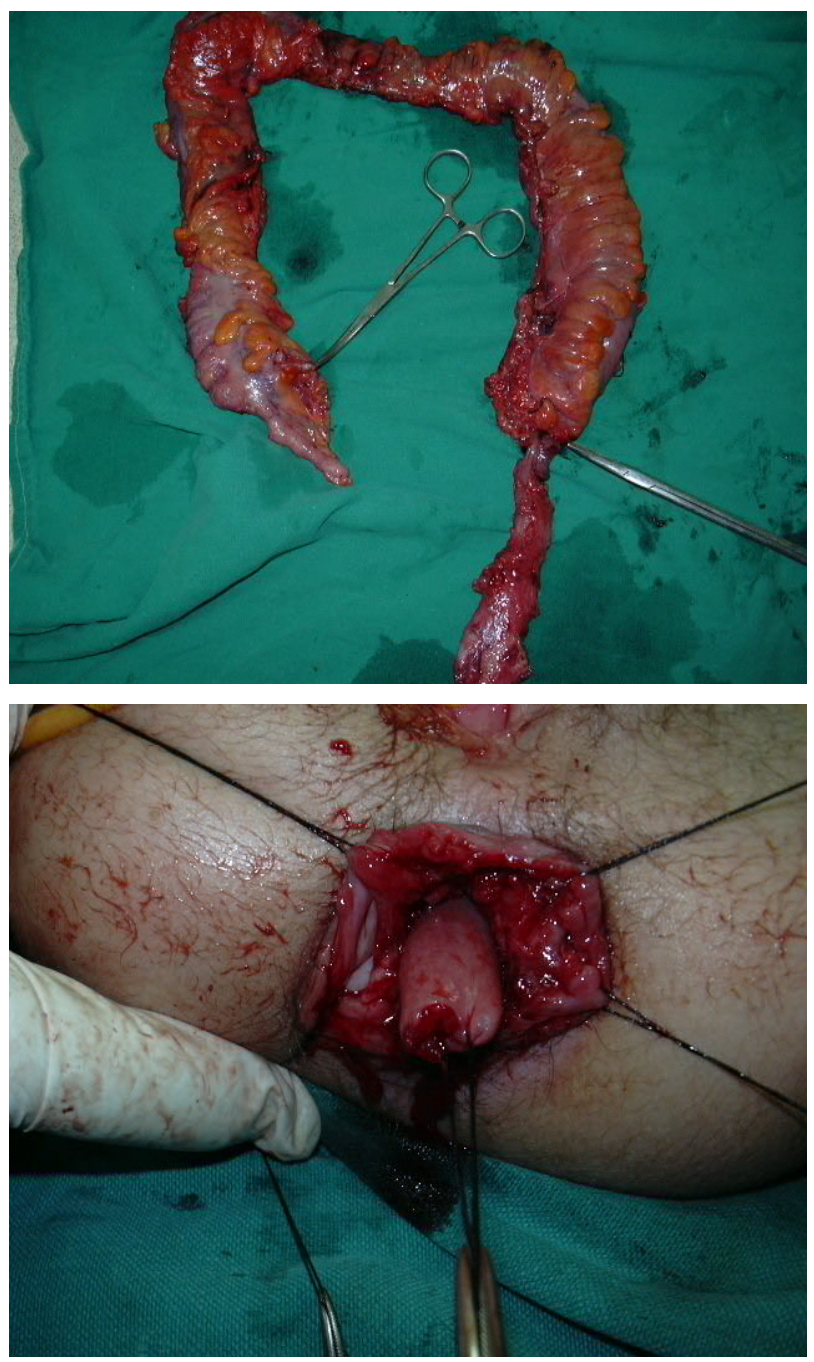

Figure 3. Total colectomy with ileoanal pull through.

mucoid carcinoma.

Prophylactic stoma was preformed in 6 patients in CAP and 9 patients of LAR. A closure of the diverting stoma could be performed, on average, 18 weeks after surgery. The closure rates were significantly lower in patients with anastomotic leakage. In one patient with closure of the colostomy and weak functional results, and in one patient with recto-vaginal fistula, a permanent colostomy was again performed after previous closure.

Major leakage occurs in 2 case of colo-anal pull through due to ischemia of rectal stump and complete retraction of the stump requiring refashioning in one case and reanastomosis in the other case and covering ileostomy.

One case of minor leakage managed conservatively. For low anterior resection 1 case of major leakage managed by covering ileostomy and 3 cases of minor leakage managed conservatively.

Only one case with covering stoma develop minor leakage which managed conservatively all case of continent perineal colostomy developed sever stenosis and fi- brosis with poor continence requiring reconstruction of terminal colostomy.

With a median follow-up of 26 months (range $4-72$ months) from the start of RT, $10(20.4 \%)$ patients have died from their disease 4 in colo-anal pullthrough group 4 in low anterior resection group and 2 in continent perineal colostomy. thirteen $(26.5 \%)$ of 49 patients exhibited tumor progression. Nine (18.3\%) developed local recurrence; 4 in CAP group 4 in LAR group and 1 in CPC group. Four patients had progress of disease without local recurrence 2 in CAP patients 1 LAR and 1 in CPC.

Tumor progression was diagnosed at an average of 20 months (4 - 60 months).

The 4-year actuarial survival for all patients was $69 \%$.

The 2-year actuarial locoregional control rates were $76.5 \%$ for all patients, $86 \%$ for the 14 patients with marked pathological tumour response to chemoradiation.

No significant difference in disease-free survival was observed between patients operated by LAR, Continent perineal colostomy or by Trans anal pull through surgery.

The characteristics surgical types, surgical complication and functional outcome of patients (Table 2).

\section{Disscussion}

Historically, patients with mid to distal rectal cancers had to undergo abdominoperineal resection to achieve adequate oncological clearance. However, with better equipment and improved surgical techniques, low anterior resection with a low colorectal or coloanal anastomosis has become the technique of choice [10]. Two major issues encountered in the surgical resection of low rectal cancers (tumor located $<6 \mathrm{~cm}$ from anal verge) are tumorfree surgical resection margin and adequate fields of colo-anal pull-through anastomosis [11].

The beneficial effect of preoperative pelvic irradiation as an adjuvant treatment has been confirmed in several studies [12,13].

Preoperative radiochemotherapy decreases the tumor volume, induces downstaging, and facilitates surgical resection. In addition, it transforms the vegetative component of the tumor into an ulcerative scar, and this may decrease intraoperative tumor seeding [14]. We observed significant tumor regression $47 \%$, This high rate of tumor regression, compared with the $18 \%$ to $26 \%$ reported after preoperative radiotherapy alone $[15,16]$, is similar to the $53 \%$ to $64 \%$ observed after preoperative radiotherapy potentiated by chemotherapy [17-19].

Adding chemotherapy to preoperative radiotherapy improve both local control and distal disease [14]. The median age of our study is 46 years which younger than age reported in other studies, where they reported median age range from 59 - 70 years the exact cause cannot be explained but may be due to genetic background or exposure to carcinogenic materials. 
Table 2. Sphincter-saving procedure, surgical complication and functional outcome.

\begin{tabular}{|c|c|c|c|}
\hline & Colo anal pull through (COP) & $\begin{array}{l}\text { LAR (low anterior } \\
\text { resection) }\end{array}$ & $\begin{array}{l}\text { Continent perineal } \\
\text { colostomy }\end{array}$ \\
\hline \multicolumn{4}{|l|}{ Gender } \\
\hline Male & $12(63.1 \%)$ & $11(50 \%)$ & $4(100 \%)$ \\
\hline Female & $7(36.9 \%)$ & $11(50 \%)$ & 0 \\
\hline Age range & $23-62$ years & $31-70$ years & $35-55$ years \\
\hline median age & 42 years & 48 years & 43 years \\
\hline Procedure & Colo-anal pull through 18 & Stapler 9 & \\
\hline Frocedure & Ileo-anal pull through 1 & Hand swing 13 & \\
\hline \multicolumn{4}{|l|}{ Leakage } \\
\hline - Major & 2 (complete detachment $\&$ retracted & 1 & \\
\hline & intra-abdominally) & & No \\
\hline - Minor & 1 & 3 & \\
\hline Prophylactic stoma & 6 & 9 & \\
\hline - Colostomy & 5 & 5 & No \\
\hline - Ileostomy & 1 & 4 & \\
\hline Postoperative stenosis & $5(26 \%)$ & $4(18 \%)$ & $4(100 \%)$ \\
\hline Urologic Complication & $3(15.8 \%)$ & $1(4.5 \%)$ & No \\
\hline \multicolumn{4}{|l|}{ Disease progression } \\
\hline - Local recurrence & $3(15.8 \%)$ & $2(9 \%)$ & 0 \\
\hline - Distance metastasis & $1(5.3 \%)$ & $3(13.6 \%)$ & 1 \\
\hline - Local and distance metastasis & $1(5.3 \%)$ & $1(4.5 \%)$ & 0 \\
\hline \multicolumn{4}{|l|}{ Functional outcome } \\
\hline $\begin{array}{l}\text { - Continence for solid \& liquid } \\
\text { stool \& flatus }\end{array}$ & $8(42.1 \%)$ & $15(68.2 \%)$ & 0 \\
\hline $\begin{array}{l}\text { - Continence for solid stool \& occasional } \\
\text { incontinence for liquid stool }\end{array}$ & $5(26.3 \%)$ & $5(22.7 \%)$ & 1 \\
\hline - Soiling at night & $2(10.6 \%)$ & $1(4.5 \%)$ & 2 \\
\hline $\begin{array}{l}\text { - Frequent episodes of incontinence } \\
\text { for liquid stool }\end{array}$ & $2(10.6 \%)$ & $1(4.5 \%)$ & 1 \\
\hline
\end{tabular}

Most patients with rectal carcinoma can now be treated by sphincter-saving surgery, but many pay a price for restoration of continuity. The "anterior resection syndrome" of faecal leakage and urgency of defaecation may afflict up to 50 per cent of patients after low anterior resection and is more common after very low colorectal or coloanal anastomosis [20].

The recurrence rate in our study is $18.3 \%$ of the curatively operated patients is in accordance with other studies of this surgical procedure, where rates range from 0 to $30 \%$ [21-25].

The method of anastomosis (hand-sewn vs. stapled) does not seem to influence recurrence rates [26].

Problems concerning the anastomosis, mostly insufficiencies, present the most common and widely discussed complications in rectal surgery. The depth of the anas- tomosis and the aim of a tension-free anastomosis in the pull-through operation make high demands on the blood supply of the colon. Most likely, the blood supply suffers from the mobilization and the skeletonization of the mesocolon.

Suture insufficiency plays a key role in postoperative morbidity. The rate of this complication after CAA is generally reported to be comparable with previous study [21-23,27]. The correlation of this complication and neoadjuvant radiation (most likely by causing obliterating endarteritis) was significant.

Stricture formation, a common problem at the site of the anastomosis, occurred in 13 patients $26.5 \%$, (five in CAP and 4 of LAR and in 4 patients of CPC) in the patients in this study. This rate does not clearly differ from the rates after deep anterior resection, although not much 
data exists for comparison. In general, this complication is considered to be higher in stapled anastomosis [28].

The fistula formation was encountered in $1(2 \%)$ patient developed rectovaginal fistula after LAR using stapler techniques.

After deep anterior resection with stapled anastomosis, the rate of fistula formation was approximately $2 \%$ [29]. In addition to rectal and gynecological surgery, radiation and chemotherapy can typically lead to fistula formation [30,31].

Acceptable anorectal continence after rectal resection and hand-sewn anastomosis is widely described in the literature [21,23,32-37].

In most of these studies the majority of patient continence was described as low grade (Grade I or II according to Kirwan). The overall disillusioning results in this study in comparison to other studies have to be analyzed critically, as a uniform classification was not used in every study, and all patients in this study had reduced sphincter function caused by treatments prior to surgery. Generally, a hand-sewn suture facilitates nerve growth through the anastomosis, which is more likely to be impaired by stapled anastomosis, and can lead to better discrimination and a better recovery of the anal inhibitory reflex [38]. Presurgical radiotherapy of the rectum or its neighboring organs further influences anorectal continence [27]. However the neoadjuvany therapy downstage the locally advanced low rectal cancer and change the decision option from abdomenoperineal to sphincter saving procedures in many cases.

\section{Conclusions}

There is a tendency of rectal cancers to affect younger groups. Most patients presented advanced stage and aggressive histopathology.

Using a multimodal approach, conservative treatment was possible in patients with locally advanced carcinomas of the lower third of the rectum that would have required APR in most instances. Preoperative radiochemotherapy was associated with a low rate of complications and induced significant down staging, Coloanal pullthrough technique is not a widely spread technique for low rectal cancer with good oncological safety and acceptable functional outcome however large numbers of patients have to study.

\section{Acknowledgements}

The authors received no financial or other support for the research reported in manuscript.

Contributions: M.A.S. participate in collection of patient's data, patient diagnoses and surgical evaluation and surgical resection of patient subjected to surgery, general coordination, drafting of manuscript and writing final manuscript.

H.A. Carried out collection of patient's data, patient diagnoses, radiotherapy administration, follow up. A.I. carried out collection of patient's data, patient diagnoses, chemotherapy administration and follow up. G.A. And A.W. participate in surgical evaluation and management of patients subjected to surgery. All authors have read and approved manuscript.

\section{REFERENCES}

[1] R. P. Billingham, "Conservative Treatment of Rectal Cancer. Extending the Indications," Cancer, Vol. 70, No. 5, 1992, pp. 1355-1363. doi:10.1002/1097-0142(19920901)70:3+<1355::AID-CN CR2820701525>3.0.CO;2-9

[2] L. D. MacDonald, "The Health of Rectal Cancer Patients in the Community," European Journal of Surgical Oncology, Vol. 11, No. 3, 1985, p. 235.

[3] A. Abdelsalem and Y. A. K. Ismail, "Preoperative Radiochemotherapy in Locally Advanced Rectal Cancer," Journal of the Egyptian National Cancer Institute, Vol. 13, No. 2, 2008, pp. 101-108.

[4] C. V. Allen, "A Pilot Study on Preoperative Irradiation of Rectosigmoid Carcinoma," American Journal of Roentgenology, Radium Therapy, and Nuclear Medicine, Vol. 114, No. 3, 1972, pp. 504-508. doi:10.2214/ajr.114.3.504

[5] J. A. Martenson Jr., et al., "Radiation Therapy Quality Control in a Clinical Trial of Adjuvant Postoperative Treatment for Rectal Cancer," International Journal of Radiation Oncology Biology Physics, Vol. 32, No. 1, 1995, pp. 51-55. doi:10.1016/0360-3016(95)00526-5

[6] J. Papillon, et al., "Role of Preoperative Radiotherapy in Preservation of the Anal Sphincter in Cancer of the Lower Rectum," Bulletin du Cancer, Vol. 77, No. 1, 1990, pp. 15-21.

[7] G. Marks, M. Mohiuddin and L. Masoni, "The Reality of Radical Sphincter Preservation Surgery for Cancer of the Distal $3 \mathrm{~cm}$ of Rectum Following High-Dose Radiation," International Journal of Radiation Oncology Biology Physics, Vol. 27, No. 4, 1993, pp. 779-783. doi:10.1016/0360-3016(93)90449-6

[8] P. Rouanet, "Sphincter Conservation and Cancer of the Lower Rectum: Argument for a Multicenter Prospective Study for Conservation of the Sphincter after Irradiation," Bulletin du Cancer, Vol. 85, No. 4, 1998, pp. 328-332.

[9] R. J. Heald, "Recurrence and Survival after Total Mesorectal Excision for Rectal Cancer," Lancet Oncology, Vol. 1, No. 8496, 1986, pp. 1479-1482.

[10] W. S. Tan, et al., "Meta-Analysis of Defunctioning Stomas in Low Anterior Resection for Rectal Cancer," British Journal of Surgery, Vol. 96, No. 5, 2009, pp. 462-472. doi:10.1002/bjs.6594

[11] Y. S. Yeh, et al., "Transanal Inside-Out Rectal Resection for Ultra-Low Rectal Cancer," Journal of Investigative Surgery, Vol. 25, No. 6, 2012, pp. 375-380. doi: $10.3109 / 08941939.2012 .655369$ 
[12] O. Dahl, et al., "Low-Dose Preoperative Radiation Postpones Recurrences in Operable Rectal Cancer. Results of a Randomized Multicenter Trial in Western Norway," Bulletin du Cancer, Vol. 66, No. 11, pp. 2286-2294. doi:10.1002/1097-0142(19901201)66:11<2286::AID-CN CR2820661106>3.0.CO;2-T

[13] L. Pahlman, "Pre- or Postoperative Radiotherapy in Rectal and Rectosigmoid Carcinoma. Report from a Randomized Multicenter Trial," Annals of Surgical Oncology, Vol. 211, No. 2, 1990, pp. 187-195. doi:10.1097/00000658-199002000-00011

[14] E. Rullier, et al., "Preoperative Radiochemotherapy and Sphincter-Saving Resection for T3 Carcinomas of the Lower Third of the Rectum," Annals of Surgery, Vol. 234, No. 5, 2001, pp. 633-640. doi:10.1097/00000658-200111000-00008

[15] C. Berger, et al., "Preoperative Radiotherapy (RT) for Rectal Cancer: Predictive Factors of Tumor Downstaging and Residual Tumor Cell Density (RTCD): Prognostic Implications," International Journal of Radiation Oncology Biology Physics, Vol. 37, No. 3, 1997, pp. 619-627. doi:10.1016/S0360-3016(96)00577-9

[16] M. C. Kaminsky-Forrett, et al., "Prognostic Implications of Dowstaging Following Preoperative Radiation Therapy for Operable T3-T4 Rectal Cancer," International Journal of Radiation Oncology Biology Physics, Vol. 42, 1998, pp. 935-941. doi:10.1016/S0360-3016(98)00345-9

[17] T. A. Rich, J. A. Ajani, et al., "Preoperative Infusional Chemoradiation Therapy for Stage T3 Rectal Cancer," International Journal of Radiation Oncology Biology Physics, Vol. 32, 1995, pp. 1025-1029. doi:10.1016/0360-3016(95)00020-Y

[18] V. Valentini, N. Cellini, et al., "Preoperative Chemoradiation for Extraperitoneal T3 Rectal Cancer: Acute Toxicity, Tumor Response, and Sphincter Preservation," International Journal of Radiation Oncology Biology Physics, Vol. 40, 1998, pp. 1067-1075.

[19] N. A. Janjan, J. Abbruzzese, et al., "Tumor Downstaging and Sphincter Preservation with Preoperative Chemoradiation in Locally Advanced Rectal Cancer: The M. D. Anderson Cancer Center Experience," International Journal of Radiation Oncology Biology Physics, Vol. 44, 1999, pp. 1027-1038. doi:10.1016/S0360-3016(99)00099-1

[20] A. S. Miller, et al., "Factors That Influence Functional Outcome after Coloanal Anastomosis for Carcinoma of the Rectum," British Journal of Surgery, Vol. 82, No. 10, 1995, pp. 1327-1330. doi:10.1002/bjs. 1800821010

[21] D. Benchimol, et al., "Oncological and Functional Results of Direct Colo-Anal Anastomosis after Total Resection of the Rectum for Cancer," Annales De Chirurgie, Vol. 48, No. 7, 1994, pp. 596-603.

[22] B. Bittorf, et al., "Functional Outcome after Intersphincteric Resection of the Rectum with Coloanal Anastomosis in Low Rectal Cancer," European Journal of Surgical Oncology, Vol. 30, No. 3, 2004, pp. 260-265. doi:10.1016/j.ejso.2003.11.011

[23] A. G. Parks and J. P. Percy, "Resection and Sutured ColoAnal Anastomosis for Rectal Carcinoma," British Journal of Surgery, Vol. 69, No. 6, 1982, pp. 301-304. doi:10.1002/bjs. 1800690602

[24] V. Schumpelick and J. Braun, "Intersphincteric Rectum Resection with Radical Mesorectum Excision and ColoAnal Anastomosis," Chirurg, Vol. 67, No. 2, 1996, pp. 110-120.

[25] A. Kohler, et al., "Long-Term Results of Low Anterior Resection with Intersphincteric Anastomosis in Carcinoma of the Lower One-Third of the Rectum: Analysis of 31 Patients," Diseases of the Colon \& Rectum, Vol. 43, No. 6, 2000, pp. 843-850. doi:10.1007/BF02238025

[26] P. M. Sagar and J. H. Pemberton, "Surgical Management of Locally Recurrent Rectal Cancer," British Journal of Surgery, Vol. 83, No. 3, 1996, pp. 293-304. doi: $10.1002 /$ bjs. 1800830305

[27] P. Luna-Perez, et al., "Anal Sphincter Preservation in Locally Advanced Low Rectal Adenocarcinoma after Preoperative Chemoradiation Therapy and Coloanal Anastomosis," Journal of Surgical Oncology, Vol. 82, No. 1, 2003, pp. 3-9. doi:10.1002/jso. 10185

[28] G. Eichhoff, "Short- and Long-Term Results of HandSewn Coloanal Anastomosis Performed as a Salvage Procedure after Rectal Resection," The Internet Journal of Surgery, Vol. 18, No. 1, 2009, pp. 1528-8242. doi:10.1007/BF02554801

[29] H. K. Antonsen, "Early Complications after Low Anterior Resection for Rectal Cancer Using the EEA Stapling Device. A Prospective Trial," Diseases of the Colon \& Rectum, Vol. 30, 1987, pp. 579-583.

[30] S. Athanasiadis, "Surgical Treatment of Radiation-Induced Rectovaginal Fistulas by the Continence Resection Procedure," Zentralblatt fur Chirurgie, Vol. 107, 1982, pp. 1160-1168.

[31] M. P. Nowacki, "Ten Years of Experience with Parks' Coloanal Sleeve Anastomosis for the Treatment of PostIrradiation Rectovaginal Fistula," European Journal of Surgical Oncology, Vol. 17, No. 6, 1991, pp. 563-566.

[32] R. Schiessel, et al., "Intersphincteric Resection for Low Rectal Tumours," British Journal of Surgery, Vol. 81, No. 9, 1994, pp. 1376-1378. doi:10.1002/bjs.1800810944

[33] E. Rullier, et al., "Laparoscopic Intersphincteric Resection with Coloplasty and Coloanal Anastomosis for Mid and Low Rectal Cancer," British Journal of Surgery, Vol. 90, No. 4, 2003, pp. 445-451. doi:10.1002/bjs.4052

[34] S. H. Baik, et al., "Hand-Sewn Coloanal Anastomosis for Distal Rectal Cancer: Long-Term Clinical Outcomes," Journal of Gastrointestinal Surgery, Vol. 9, No. 6, 2005, pp. 775-780. doi:10.1016/j.gassur.2005.03.003

[35] M. R. Keighley and D. Matheson, "Functional Results of Rectal Excision and Endo-Anal Anastomosis," British Journal of Surgery, Vol. 67, No. 10, 1980, pp. 757-761. doi:10.1002/bjs. 1800671021

[36] P. Rouanet, et al., "Conservative Surgery for Low Rectal Carcinoma after High-Dose Radiation. Functional and Oncologic Results," Annals of Surgery, Vol. 221, No. 1, 1995, pp. 67-73. doi:10.1097/00000658-199501000-00008

[37] R. Schiessel, M. Wunderlich and R. Waneck, "Results of Colo-Anal Anastomosis in Deep-Seated Tumors of the 
Rectum," Chirurg, Vol. 57, No. 12, 1986, pp. 792-796.

[38] P. G. Horgan, et al., "Effect of Anterior Resection on Anal Sphincter Function,” British Journal of Surgery, Vol.
76, No. 8, 1989, pp. 783-786.

doi:10.1002/bjs. 1800760805 